\title{
Pyroptosis-Mediated Cell Death as an Approach for Novel
}

\section{Cancer Treatment}

Megan Lloren

Oxford Academy

Edward Thomas

Hinsdale South High School

Running Head: Pyroptosis-Mediated Cell Death Approaches

Precis: To understand the pathways through which cancer evades cell death and metastasis, apoptosis has been extensively researched but little has been done to understand pyroptosis. Here we review alternative ways of furthering research and understanding of cancer biology through investigating pyroptosis.

Funding Statement: None

Conflicts of Interest: None 


\section{Pyroptosis-Mediated Cell Death as an Approach for Novel}

\section{Cancer Treatment}

\section{Abstract}

Cancer is the leading cause of death in the world, which makes understanding its biology and creating new treatments to save lives vitally important. To understand the pathways through which cancer evades apoptosis and how death can be induced upon cancerous cells, apoptosis has been extensively researched, but we propose the benefits of investigating another form of mediated cell death--pyroptosis--to understand alternative ways that cancer can be treated and how utilizing pyroptosis may be a more effective alternative than apoptosis. We also review one of the most controversial usages of bacteria-mediated treatment for cancer, as well as advise future researchers hoping to implement pyroptosis-based treatment to investigate the necessity of the situation before resorting to bacteria-mediated onco-treatments. 


\section{Introduction}

The result of genetic alterations and uncontrolled growth of mutated cells (Sporn, 1996), cancer is the leading cause of death worldwide (Mahase, 2019; Heron, 2016.; Twombly, 2005; see: Sidney et. al., 2019; Hastings et al., 2018), causing nearly 10,000 individuals to die in 2020 alone (Ferlay et. al., 2020). Misregulation in genetic transcription appears to be the primary cause of carcinogenesis in cells (Lee \& Young, 2013; see Jones \& Baylin, 2007) despite efforts of the body to prevent such errors (see Anagnostou et. al., 2021). These cancerous cells evade detection by the immune system (Loose \& Van de Wiele, 2009; Corthay, 2014; Cavallo et. al., 2011; Prestwich et. al., 2008) and continue to grow into tumors.

Due to its lethality (Shen et. al., 2020), current treatment methods depend largely on radiation therapy, chemotherapy, or surgery (Hanna et. al., 2020) to detect cancer biomarkers, remove or control tumor growth (Zhang et. al., 2020). However, various disseminated cancers can evade traditional treatment options, being therefore resistant to the current options and continue to metastasize in the body (Gottesman, 2002). Because of this, immunotherapy and bacterial therapy approaches are being utilized (Olino et. al., 2020) and have been found to be safer and possibly more effective than traditional methods (eg. oncolytic virus-mediated cancer cell death, genetic counseling, and cytotoxic therapy (Ma et. al., 2020; see Hussein, 2002).

Current therapies rely on stopping the proliferation of rapidly-dividing cells (National Cancer Institute, "Chemotherapy to Treat Cancer", 2015), shrinking tumors (National 
Cancer Institute, "Chemotherapy to Treat Cancer", 2015; National Cancer Institute, "Hormone Therapy to Treat Cancer", 2015; National Cancer Institute, "Hyperthermia to Treat Cancer", n.d.), preventing the recurrence of cancer (National Cancer Institute, "Hormone Therapy to Treat Cancer", 2015), increase the immune system's response/sensitivity against cancer (National Cancer Institute, "Cancer Treatment Vaccines", 2019; National Cancer Institute, “Immune Checkpoint Inhibitors", 2019; National Cancer Institute, "Immune System Modulators", 2019; National Cancer Institute, "Monoclonal Antibodies", 2019; National Cancer Institute, "T-cell Transfer Therapy", n.d.), using other viruses/bacteria (National Cancer Institute, "Cancer Treatment Vaccines", 2019), sensitizing cancer cells to treatment (National Cancer Institute, "Photodynamic Therapy for Cancer", n.d.), damaging the DNA of cancer cells (National Cancer Institute, "Radiation Therapy for Cancer", n.d.), or tumor removal (National Cancer Institute, "Surgery for Cancer", 2015) to weaken and kill cancer -oftentimes through apoptosis.

Apoptosis is the programmed death of cellular entities in normal cell turnover and death to maintain cell populations and prevent the survival of cells with damaged or altered DNA (Elmore, 2007) and is a key part of the body's natural defenses against carcinogenesis in cells. Because apoptosis is dysregulated in many cancers, it has been particularly investigated in recent years (Reed, 2000) through and that, by investigating the mechanisms cancer takes to evade apoptosis, apoptosis can be force-induced in cancerous cells and metastasis can be prevented. One of these methods was approved by the Food and Drug Administration (FDA) as a viable 
treatment option for some cases of melanoma, using proteasome inhibitor bortezomib as a catalyst for synergistic levels of apoptosis (Nawrocki et. al., 2006).

However, because of the complexities in cancer biology and variety of methods of evading apoptosis that vary even within the same form of cancer as is the hallmarks of cancer (Floor et. al., 2012), other, newer avenues to understand the pathophysiology of said disease and develop new therapies are being explored (Floor et. al., 2012) -- one such avenue being exploring pyroptosis due to its efficacy and speed, as compared to apoptosis (Wang, Jiang et. al., 2019), and of its ability to inhibit tumor growth with the usage of the Gasdermin family (Xia et. al., 2019). This paper reviews the importance of research into pyroptosis as a new model of understanding cancer biology and important considerations and obstacles in bringing pyroptosis research into the "cancer-research spotlight".

\section{Mechanisms of Pyroptosis}

Although apoptosis and pyroptosis describe a form of programmed cell death regulated by proteins (Tsuchiya, 2020), pyroptosis specifically works through inflammatory and necrotic cell death (Robinson et. al., 2019; Fang et. al., 2020; McKenzie et.al., 2020) through the triggering of specific inflammasomes -- including the nod-like receptor (NLR) family, the DNA receptor Absent in Melanoma 2 (AIM2), and the Pyrin receptor (Bertheloot et. al., 2021)-- by the activation caspases 1, 3, 4, 5, and 11 (found in mice) (Man et.al, 2017), which therefore cleaves Gasdermin D and E, allowing for the release of said inflammasomes: cytokinetic proteins (Fang et. al., 2020; McKenzie et.al., 2020). 
Cytokinetic proteins then will promote cytokinesis, which researchers have discovered to its association to carcinogenesis and modulated cell death (Sagona \& Stenmark, 2010).

As for the pathways by which pyroptosis occurs, scientists consider activation through caspase- 1 canonical and activation through caspases 3,5 , and 11 as non-canonical, based on which inflammasomes are released (Zheng, Liwinski, \& Elinav, 2020). Upon the activation of caspase-1 within the canonical inflammasome, compared to the activation through caspases- $3,4,5$, and 11 within the non-canonical inflammasome of cytosolic lipopolysaccharide (LPS), caspase-1 activation promotes pyroptosis through the release of pro-inflammatory cytokinetic interleukin (IL)-1 $1 \beta$ and IL-18 and signals to cleave Gasdermin D, which will then encourage pyroptosis to occur (Evavold et al, 2018). In comparison, in the non-traditional and non-canonical approach, caspase 3,4 , 5 , and 11 skips the release of the cytokinetic interleukin and goes straight to the cleaving the Gasdermin D but trigger a second inflammasome to release cytokinesis (Swanson et al, 2019).

\section{Pyroptosis versus Apoptosis}

While both pyroptosis and apoptosis induce programmed cellular death (Zheng \& $\mathrm{Li}$, 2020), maintain homeostasis (Zheng \& Li, 2020), destroy DNA (Fang et. al., 2020), and carry out nuclear condensation and caspase independence (Fang et. al., 2020), the apoptotic cellular process differs as it is considered non-inflammatory and is not mediated by the plasma membrane (Zhang et al, 2018). The apoptotic cellular process 
has two main pathways, with the extrinsic signaling through death receptors or intrinsic through the mitochondria (Xu \& Shi, 2007; Elmore, 2007). Though both pyroptosis and apoptosis can be activated through caspase-1, pyroptosis also depends on the activation of caspases-4, 5, and 11 (Fang et. al., 2020). Interestingly, because pyroptosis has far fewer steps in the process of cell death (He et al, 2018) than apoptosis, pyroptotic cell death decreases the likelihood of procedural errors to lead to carcinogenesis and metastasis (Gong et al., 2019).

The comparative benefits of pyroptosis versus apoptosis even extend to current cancer treatments, with more tumors becoming more resistant to the most recent chemotherapeutic treatments focused on downregulating tumors through apoptotic pathways (Ju et al., 2021). Recent studies suggest combining pyroptosis with other forms of mediated cell death to combat multi-drug-resistant tumors (Long et al., 2021). Because of this, pyroptosis presents itself as a particularly useful area of research into pathways of forcing cell death in cancerous cells.

\section{Gasdermin D and E}

Gasdermin D and E (GSDMD and GSDME) in themselves modulate the cell proliferation and differentiation within an organism, which then will promote the process of pyroptosis (Li et. al., 2020). After inflammasomes activate caspase-1, caspase-1 cleaves GSDMD, a major player in the pyroptotic process in various cancers (Fang et. al., 2020). GSDMD regulates when pyroptosis will occur, while in comparison, GSDME will promote pyroptosis through cleavage of GSDME then activates the caspase 
cascade through the activation of caspase-3 (Ibrahim et.al., 2021). Through the activation of caspase- 3 , rather than the activation of caspase-8 in apoptosis, the focus of utilizing GSDME to pyroptosis has the potential of preserving already healthy cells, noted through a recent study conducted through cultured human renal tubular epithelial cells and its preservation through induced nephrotoxicity as a result of cleaving caspase-3 and GSDME (Shen et.al., 2021). However, pyroptotic-induced cell death may only occur if activated with GSDME to form pores within the cellular membrane (Ramos-Junior, E. S., \& Morandini, A. C. (2017). Gasdermin: A new player to the inflammasome game. biomedical journal, 40(6), 313-316).

The presence of GSDME has also proven to be one of the hallmarks of current malignant tumors and cancers, such as melanoma and lung cancer, since GSDME was found to be up-related in these areas (Wang, Liu, \& Zhao, 2019). Researchers found that these noticeable levels of GSDME could be used as a prognostic indicator for cancers, and besides its potential in promoting the process of pyroptosis through said overexpression of that given protein (Wang, Liu, \& Zhao, 2019; Yu et al, 2021). 


\section{Discussions:}

\section{The Ethical Dilemma of Implementing Pyroptosis}

Newer approaches to cancer come with newer ethical dilemmas associated with these treatments (Stoeklé et al., 2018). Recent efforts to implement pyroptosis via bacteria implantation to induce an immune system response to a glioblastoma multiform tumor to, in theory, prevent tumor metastasis, was heavily criticized in the medical community (Swazo, n.d.) -- even featured in the New Yorker in 2015 (Eakin, 2015). While this attempt through bacterial implantation did not work, a similar theory was hypothesized by later researchers by oncolytic virus-based treatments (Ma et. al., 2020). However, these treatments present similar issues as bacteria-based treatments, many of which concern safety and post-treatment side effects (Fu et.al., 2019). Firstly, how do we ensure that new technologies in cancer research are unable to cause collateral damage? And, once such technologies are developed, at what point do we transition to humans? When adding external biological organisms into a body, how do we ensure that the chance of these organisms causing an infection is minimal to nonexistent? Though these questions have been investigated in prior situations with new drugs or therapies, the introduction of bacteria designed to directly kill select bacteria will require a re-analysis and reconsideration of the ethical, moral, social, and scientific perspectives and situations with such a treatment.

\section{Multi-Drug Resistant Cancers}

Yet another important consideration when developing and implementing pyroptosis-based cancers is ensuring that cancers do not become resistant to pyroptotic 
drugs, and therefore require alternative and possibly harsher treatments (see Mansoori et al., 2017). All treatments against cancer have the possibility of becoming ineffective or less effective against cancers as the cancers develop mechanisms to decrease drug absorption, repair intercellular systems, or evade drug-induced apoptosis (Gillet \& Gottesman, 2010), but how might we develop pyroptosis-based technologies in such a way that cancers are prevented or limited from evolving to evade cell death like other traditional therapies?

\section{Lack of Research into Pyroptosis, Necroptosis, and other Forms of Cell Death}

Current research has identified three main forms of mediated cell death -- Apoptosis, Pyroptosis, and Necroptosis (Liu et al., 2018; Green \& Llambi, 2015; Tan et al., 2021) -and investigations continue in these field areas in hopes of better understanding how cancerous cells evade cell death. Apoptosis was long considered the sole form of cell death until 1972 (Fink \& Cookson, 2005) when pyroptosis and necroptosis were found in 1992 (Yu et al, 2021) and although understanding the mechanisms of apoptosis may help us understand how cancer cells evade the most traditional pathway of cell death, it's also vitally important for research to focus on pyroptosis and necroptosis to understand alternative ways that cancer may resist cell death and to find new forms of treatment that utilize pyroptotic and necroptotic cell death; only with further examination and exploration into other avenues of cell death will we have a complete understanding of cancer pathophysiology and mechanisms. Additionally, there may be the possibility of other forms of cell death that have yet to be discovered (Koren \& Fuchs, 2021) that may hold the key to understanding and effectively killing cancer cells. 


\section{Conclusion}

Research into cancer biology and mechanisms is crucial to the advancement of our understanding of carcinogenesis, metastasis, and treatment. One current focus in the field is understanding the pathways of apoptosis and how apoptosis can be forcefully triggered within the cell to prevent the metastatic spread of cancer and deliver treatments effectively. However, because pyroptosis is becoming a more promising option, with new research, it's crucial to aim future research efforts towards understanding the mechanisms of pyroptosis as an alternative method of finding novel cancer treatment. 


\section{Acknowledgments}

We humbly thank Dr. Jalees Rehman from the University of Illinois in Chicago for his guidance, support, and inspiration throughout this paper. His work into pyroptosis inspired us to create this literature review in hopes of summarizing the diverse research focuses in the field and encouraging future researchers to investigate pyroptosis as a pathway of understanding cancer biology. 


\section{Conflict of Interest}

We claim no conflict of financial, commercial, or personal interest in writing this paper. 


\section{Bibliography}

Anagnostou, M. E., Chung, C., McGann, E., Verheijen, M., Kou, Y., Chen, L., \& Vermulst, M. (2021). Transcription errors in aging and disease. Translational Medicine of Aging.Chicago

Bertheloot, D., Latz, E., \& Franklin, B. S. (2021). Necroptosis, pyroptosis and apoptosis: an intricate game of cell death. Cellular \& Molecular Immunology, 18(5), 1106-1121.

Cancer Treatment Vaccines - Immunotherapy. (2019, September 24). National Cancer Institute.

https://www.cancer.gov/about-cancer/treatment/types/immunotherapy/cancer-treatme nt-vaccines

Cavallo, F., De Giovanni, C., Nanni, P., Forni, G., \& Lollini, P. L. (2011). 2011: the immune hallmarks of cancer. Cancer Immunology, Immunotherapy, 60(3), 319-326.

Chemotherapy to Treat Cancer. (2015, April 29). National Cancer Institute. https://www.cancer.gov/about-cancer/treatment/types/chemotherapy

Corthay, A. (2014). Does the immune system naturally protect against cancer?. Frontiers in immunology, 5, 197.Chicago

Eakin, E. (2015, November 30). Bacteria on the Brain. The New Yorker. https://www.newyorker.com/magazine/2015/12/07/bacteria-on-the-brain

Elmore, S. (2007). Apoptosis: a review of programmed cell death. Toxicologic pathology, 35(4), 495-516.

Evavold, C. L., Ruan, J., Tan, Y., Xia, S., Wu, H., \& Kagan, J. C. (2018). The Pore-Forming Protein Gasdermin D Regulates Interleukin-1 Secretion from Living 
Macrophages. Immunity, 48(1), 35-44.e6.

https://doi.org/10.1016/j.immuni.2017.11.013

Fang, Y., Tian, S., Pan, Y., Li, W., Wang, Q., Tang, Y., ... \& Shu, Y. (2020). Pyroptosis: a new frontier in cancer. Biomedicine \& Pharmacotherapy, 121, 109595.

Ferlay J, Ervik M, Lam F, Colombet M, Mery L, Piñeros M, et al. Global Cancer Observatory: Cancer Today. Lyon: International Agency for Research on Cancer; 2020 (https://gco.iarc.fr/today, accessed February 2021

Fink, S. L., \& Cookson, B. T. (2005). Apoptosis, pyroptosis, and necrosis: mechanistic description of dead and dying eukaryotic cells. Infection and immunity, 73(4), 1907-1916. https://doi.org/10.1128/IAI.73.4.1907-1916.2005

Floor, S. L., Dumont, J. E., Maenhaut, C., \& Raspe, E. (2012). Hallmarks of cancer: of all cancer cells, all the time?. Trends in molecular medicine, 18(9), 509-515.

Fu, L. Q., Wang, S. B., Cai, M. H., Wang, X. J., Chen, J. Y., Tong, X. M., ... \& Mou, X. Z. (2019). Recent advances in oncolytic virus-based cancer therapy. Virus research, 270, 197675.

Gillet, J. P., \& Gottesman, M. M. (2010). Mechanisms of multidrug resistance in cancer. Methods in molecular biology (Clifton, N.J.), 596, 47-76. https://doi.org/10.1007/978-1-60761-416-6_4

Gong, Y. N., Crawford, J. C., Heckmann, B. L., \& Green, D. R. (2019). To the edge of cell death and back. The FEBS journal, 286(3), 430-440. https://doi.org/10.1111/febs.14714

Gottesman, M. M. (2002). Mechanisms of cancer drug resistance. Annual review of medicine, 53(1), 615-627.Chicago 
Green, D. R., \& Llambi, F. (2015). Cell Death Signaling. Cold Spring Harbor perspectives in biology, 7(12), a006080. https://doi.org/10.1101/cshperspect.a006080 Hanna, T. P., King, W. D., Thibodeau, S., Jalink, M., Paulin, G. A., Harvey-Jones, E., ... \& Aggarwal, A. (2020). Mortality due to cancer treatment delay: systematic review and meta-analysis. bmj, 371.Chicago

Harding, M. C., Sloan, C. D., Merrill, R. M., Harding, T. M., Thacker, B. J., \& Thacker, E. L. (2018). Transitions From Heart Disease to Cancer as the Leading Cause of Death in US States, 1999-2016. Preventing chronic disease, 15, E158. https://doi.org/10.5888/pcd15.180151

Hastings, K. G., Boothroyd, D. B., Kapphahn, K., Hu, J., Rehkopf, D. H., Cullen, M. R., \& Palaniappan, L. (2018). Socioeconomic Differences in the Epidemiologic Transition From Heart Disease to Cancer as the Leading Cause of Death in the United States, 2003 to 2015: An Observational Study. Annals of internal medicine, 169(12), 836-844. https://doi.org/10.7326/M17-0796

He, B., Shi, Y., Liang, Y. et al. Single-walled carbon-nanohorns improve biocompatibility over nanotubes by triggering less protein-initiated pyroptosis and apoptosis in macrophages. Nat Commun 9, 2393 (2018). https://doi.org/10.1038/s41467-018-04700-z Heron M, Anderson RN. Changes in the leading cause of death: Recent patterns in heart disease and cancer mortality. NCHS data brief, no 254. Hyattsville, MD: National Center for Health Statistics. 2016.

Hormone Therapy for Cancer. (2015, April 29). National Cancer Institute. https://www.cancer.gov/about-cancer/treatment/types/hormone-therapy 
Hussein, M. A. (2002). Nontraditional cytotoxic therapies for relapsed/refractory multiple myeloma. The Oncologist, 7, 20-29.Chicago

Hyperthermia to Treat Cancer. (2021, June 17). National Cancer Institute.

https://www.cancer.gov/about-cancer/treatment/types/hyperthermia

Ibrahim, J., De Schutter, E., \& de Beeck, K. O. (2021). GSDME: a potential ally in cancer detection and treatment. Trends in Cancer.

Immune Checkpoint Inhibitors. (2019, September 24). National Cancer Institute.

https://www.cancer.gov/about-cancer/treatment/types/immunotherapy/checkpoint-inhi bitors

Immune System Modulators for Cancer Therapy. (2019, September 24). National Cancer Institute.

https://www.cancer.gov/about-cancer/treatment/types/immunotherapy/immune-syste m-modulators

Jones, P. A., \& Baylin, S. B. (2007). The epigenomics of cancer. Cell, 128(4), 683-692. https://doi.org/10.1016/j.cell.2007.01.029

Ju, X., Yang, Z., Zhang, H., \& Wang, Q. (2021). Role of pyroptosis in cancer cells and clinical applications. Biochimie, 185, 78-86. https://doi.org/10.1016/j.biochi.2021.03.007

Koren, E., \& Fuchs, Y. (2021). Modes of Regulated Cell Death in Cancer. Cancer discovery, 11(2), 245-265. https://doi.org/10.1158/2159-8290.CD-20-0789 Lee, T. I., \& Young, R. A. (2013). Transcriptional regulation and its misregulation in disease. Cell, 152(6), 1237-1251. https://doi.org/10.1016/j.cell.2013.02.014 
Li, L., Li, Y., \& Bai, Y. (2020). Role of GSDMB in Pyroptosis and Cancer. Cancer management and research, 12, 3033.Chicago

Liu, X., Yang, W., Guan, Z. et al. There are only four basic modes of cell death, although there are many ad-hoc variants adapted to different situations. Cell Biosci 8, 6 (2018). https://doi.org/10.1186/s13578-018-0206-6

Long, K., Gu, L., Li, L. et al. Small-molecule inhibition of APE1 induces apoptosis, pyroptosis, and necroptosis in non-small cell lung cancer. Cell Death Dis 12, 503 (2021). https://doi.org/10.1038/s41419-021-03804-7

Loose, D., \& Van de Wiele, C. (2009). The immune system and cancer. Cancer Biotherapy and Radiopharmaceuticals, 24(3), 369-376. Chicago

Ma, J., Ramachandran, M., Jin, C., Quijano-Rubio, C., Martikainen, M., Yu, D., \& Essand, M. (2020). Characterization of virus-mediated immunogenic cancer cell death and the consequences for oncolytic virus-based immunotherapy of cancer. Cell death \& disease, 11(1), 1-15.

Mahase E. (2019). Cancer overtakes CVD to become leading cause of death in high income countries. BMJ (Clinical research ed.), 366, 15368.

https://doi.org/10.1136/bmj.15368

Man, S. M., Karki, R., \& Kanneganti, T. D. (2017). Molecular mechanisms and functions of pyroptosis, inflammatory caspases and inflammasomes in infectious diseases. Immunological reviews, 277(1), 61-75.

Mansoori, B., Mohammadi, A., Davudian, S., Shirjang, S., \& Baradaran, B. (2017). The Different Mechanisms of Cancer Drug Resistance: A Brief Review. Advanced pharmaceutical bulletin, 7(3), 339-348. https://doi.org/10.15171/apb.2017.041 
McKenzie, B. A., Dixit, V. M., \& Power, C. (2020). Fiery cell death: pyroptosis in the central nervous system. Trends in neurosciences, 43(1), 55-73. Chicago

Monoclonal Antibodies. (2019, September 24). National Cancer Institute. https://www.cancer.gov/about-cancer/treatment/types/immunotherapy/monoclonal-an tibodies

Olino, K., Park, T., \& Ahuja, N. (2020, October). Exposing Hidden Targets: Combining epigenetic and immunotherapy to overcome cancer resistance. In Seminars in cancer biology (Vol. 65, pp. 114-122). Academic Press.Chicago

Photodynamic Therapy for Cancer. (2021, June 21). National Cancer Institute. https://www.cancer.gov/about-cancer/treatment/types/photodynamic-therapy

Prestwich, R. J., Errington, F., Hatfield, P., Merrick, A. E., Ilett, E. J., Selby, P. J., \& Melcher, A. A. (2008). The immune system-is it relevant to cancer development, progression and treatment?. Clinical oncology, 20(2), 101-112.Chicago

"Products - Data Briefs - Number 254 - August 2016." Centers for Disease Control and Prevention, Centers for Disease Control and Prevention, 24 Aug. 2016, www.cdc.gov/nchs/products/databriefs/db254.htm?page=7\&utm_source=hootsuite.

Radiation Therapy for Cancer. (2019, January 8). National Cancer Institute. https://www.cancer.gov/about-cancer/treatment/types/radiation-therapy

Reed, J. C. (2000). Mechanisms of apoptosis. The American journal of pathology, 157(5), 1415-1430.Chicago

Renee Twombly, Cancer Surpasses Heart Disease as Leading Cause of Death for All But the Very Elderly, JNCl: Journal of the National Cancer Institute, Volume 97, Issue 5, 2 March 2005, Pages 330-331, https://doi.org/10.1093/jnci/97.5.330 
Robinson, N., Ganesan, R., Hegedűs, C., Kovács, K., Kufer, T. A., \& Virág, L. (2019).

Programmed necrotic cell death of macrophages: Focus on pyroptosis, necroptosis, and parthanatos. Redox biology, 26, 101239.

Sagona, A. P., \& Stenmark, H. (2010). Cytokinesis and cancer. FEBS letters, 584(12), 2652-2661.

Shen, S., Vagner, S., \& Robert, C. (2020). Persistent cancer cells: the deadly survivors. Cell, 183(4), 860-874.

Shen, X., Wang, H., Weng, C., Jiang, H., \& Chen, J. (2021). Caspase

3/GSDME-dependent pyroptosis contributes to chemotherapy drug-induced nephrotoxicity. Cell death \& disease, 12(2), 1-16.

Sidney, S., Go, A. S., \& Rana, J. S. (2019). Transition From Heart Disease to Cancer as the Leading Cause of Death in the United States. Annals of internal medicine, 171(3), 225. https://doi.org/10.7326/L19-0202

Sporn, M. B. (1996, May 18). The war on cancer. The Lancet. https://www.thelancet.com/pdfs/journals/lancet/PIIS0140-6736(96)91015-6.pdf

Stoeklé, H. C., Mamzer-Bruneel, M. F., Frouart, C. H., Le Tourneau, C., Laurent-Puig, P., Vogt, G., \& Hervé, C. (2018). Molecular tumor boards: ethical issues in the new era of data medicine. Science and engineering ethics, 24(1), 307-322.

Surgery for Cancer. (2015, April 29). National Cancer Institute. https://www.cancer.gov/about-cancer/treatment/types/surgery

Swanson, K. V., Deng, M., \& Ting, J. P. (2019). The NLRP3 inflammasome: molecular activation and regulation to therapeutics. Nature reviews. Immunology, 19(8), 477-489. https://doi.org/10.1038/s41577-019-0165-0 
Swazo, N. Between "Research" and "Innovative Therapy": An Unsettled Moral Dilemma in the Muizelaar Case.

Tan, Y., Chen, Q., Li, X. et al. Pyroptosis: a new paradigm of cell death for fighting against cancer. J Exp Clin Cancer Res 40, 153 (2021). https://doi.org/10.1186/s13046-021-01959-x

T-cell Transfer Therapy - Immunotherapy. (2020, August 25). National Cancer Institute. https://www.cancer.gov/about-cancer/treatment/types/immunotherapy/t-cell-transfer-t herapy

Tsuchiya, K. (2020). Inflammasome-associated cell death: Pyroptosis, apoptosis, and physiological implications. Microbiology and immunology, 64(4), 252-269. Chicago

Wang, M., Jiang, S., Zhang, Y., Li, P., \& Wang, K. (2019). The multifaceted roles of pyroptotic cell death pathways in cancer. Cancers, 11(9), 1313.Chicago

Wang Y-Y, Liu X-L and Zhao R (2019) Induction of Pyroptosis and Its Implications in Cancer Management. Front. Oncol. 9:971. doi: 10.3389/fonc.2019.00971

Xia, X., Wang, X., Cheng, Z., Qin, W., Lei, L., Jiang, J., \& Hu, J. (2019). The role of pyroptosis in cancer: pro-cancer or pro-"host"?. Cell death \& disease, 10(9), 1-13.

Xu, G., Shi, Y. Apoptosis signaling pathways and lymphocyte homeostasis. Cell Res 17, 759-771 (2007). https://doi.org/10.1038/cr.2007.52

Yu, P., Zhang, X., Liu, N., Tang, L., Peng, C., \& Chen, X. (2021). Pyroptosis: mechanisms and diseases. Signal transduction and targeted therapy, 6(1), 128. https://doi.org/10.1038/s41392-021-00507-5 
Zhang, Y., Chen, X., Gueydan, C. et al. Plasma membrane changes during programmed cell deaths. Cell Res 28, 9-21 (2018).

https://doi.org/10.1038/cr.2017.133

Zheng, D., Liwinski, T. \& Elinav, E. Inflammasome activation and regulation: toward a better understanding of complex mechanisms. Cell Discov 6, 36 (2020). https://doi.org/10.1038/s41421-020-0167-x

Zheng, Z., \& Li, G. (2020). Mechanisms and Therapeutic Regulation of Pyroptosis in Inflammatory Diseases and Cancer. International journal of molecular sciences, 21(4), 1456. https://doi.org/10.3390/ijms21041456 